\title{
VARIABILIDADE LOCAL E REGIONAL DA EVAPOTRANSPIRAÇÃO ESTIMADA PELO ALGORITMO SEBAL
}

\author{
LUIS C. J. MOREIRA ${ }^{1}$, BRUNO J. DURAND ${ }^{2}$, ADUNIAS DOS S. TEIXEIRA ${ }^{3}$, \\ EUNICE M. ANDRADE ${ }^{4}$
}

\begin{abstract}
RESUMO: Em face da importância em conhecer a evapotranspiração (ET) para uso racional da água na irrigação no contexto atual de escassez desse recurso, algoritmos de estimativa da ET a nível regional foram desenvolvidos utilizando-se de ferramentas de sensoriamento remoto. Este estudo objetivou aplicar o algoritmo SEBAL (Surface Energy Balance Algorithms for Land) em três imagens do satélite Landsat 5, do segundo semestre de 2006. As imagens correspondem a áreas irrigadas, floresta nativa densa e a Caatinga do Estado do Ceará (Baixo Acaraú, Chapada do Apodi e Chapada do Araripe). Este algoritmo calcula a evapotranspiração horária a partir do fluxo de calor latente, estimado como resíduo do balanço de energia na superfície. Os valores de ET obtidos nas três regiões foram superiores a $0,60 \mathrm{~mm} \mathrm{~h}^{-1}$ nas áreas irrigadas ou de vegetação nativa densa. As áreas de vegetação nativa menos densa apresentaram taxa da ET horária de 0,35 a $0,60 \mathrm{~mm} \mathrm{~h}^{-1}$, e valores quase nulos em áreas degradadas. A análise das médias de evapotranspiração horária pelo teste de Tukey a 5\% de probabilidade permitiu evidenciar uma variabilidade significativa local, bem como regional no Estado do Ceará.
\end{abstract}

PALAVRAS-CHAVE: sensoriamento remoto, balanço de radiação, Landsat.

\section{LOCAL AND REGIONAL VARIABILITY OF EVAPOTRANSPIRATION ESTIMATED BY SEBAL ALGORITHM}

\begin{abstract}
In the context of water resources scarcity, the rational use of water for irrigation is necessary, implying precise estimations of the actual evapotranspiration (ET). With the recent progresses of remote-sensed technologies, regional algorithms estimating evapotranspiration from satellite observations were developed. This work aimed at applying the SEBAL algorithm (Surface Energy Balance Algorithms for Land) at three Landsat-5 images during the second semester of 2006. These images cover irrigated areas, dense native forest areas and caatinga areas in three regions of the state of Ceará (Baixo Acaraú, Chapada do Apodi and Chapada do Araripe). The SEBAL algorithm calculates the hourly evapotranspiration from the latent heat flux, estimated from the surface energy balance. The hourly evapotranspiration values obtained were greater than $0.60 \mathrm{~mm} \mathrm{~h}^{-1}$ in irrigated or dense native vegetation areas, from 0.35 to $0.60 \mathrm{~mm} \mathrm{~h}^{-1}$ in sparse vegetation areas and almost null in degradated areas. The analysis of hourly evapotranspiration means by Tukey test at 5\% probability level showed not only a significant variability locally but also at a regional scale in the state of Ceará.
\end{abstract}

KEYWORDS: remote sensing, radiation balance, Landsat.

\section{INTRODUÇÃO}

A crescente demanda hídrica e, por outro lado, a deterioração dos recursos naturais e sua escassez em algumas regiões tornam o gerenciamento integrado dos recursos hídricos cada vez mais imprescindível (SCHMIDT et al., 2004). Sabe-se que o setor agrícola é o maior consumidor de água, alcançando cerca de $69 \%$ de toda a água derivada de rios, lagos e aquíferos subterrâneos. Os

\footnotetext{
${ }^{1}$ Eng ${ }^{0}$ Agrônomo, Mestrando em Engenharia Agrícola, CCA/UFC, Caixa Postal 12168, Fortaleza - CE, cleniojario@ gmail.com.

${ }^{2}$ Eng $^{0}$ Civil, Doutorando em Engenharia Hidráulica e Ambiental, DEHA/UFC, Fortaleza - CE, brdurand@ yahoo.com.

${ }^{3}$ Eng $^{\mathrm{o}}$ Agrônomo, Ph.D. Prof. Associado, Engenharia Agrícola, CCA/UFC, adunias@ ufc.br.

${ }^{4}$ Eng $^{\mathrm{a}}$ Agrônoma, Ph.D. Profa. Associada, Engenharia Agrícola, CCA/UFC, eandrade@ pq.cnpq.br.

Recebido pelo Conselho Editorial em: 3-2-2010

Aprovado pelo Conselho Editorial em: 8-9-2010
} 
outros 31\% são consumidos pelas indústrias e uso doméstico (PAZ et al., 2000). Diante desses números, o uso racional da água na agricultura irrigada deve ser considerada uma meta prioritária.

Para quantificar a demanda hídrica de uma cultura, é necessário ter conhecimento em detalhes dos componentes do ciclo hidrológico e, principalmente, da evapotranspiração. PEREIRA et al. (1997) definem evapotranspiração como um elemento climático fundamental, composto de dois processos: a evaporação da água e a transpiração da vegetação. A evapotranspiração é fortemente influenciada pelo tipo de vegetação, pelo manejo agrícola, pela gestão ambiental e, principalmente, por parâmetros climáticos (ALLEN et al., 1998), incluindo radiação solar, vento, temperatura e umidade relativa.

Os modelos clássicos de medição da evapotranspiração fornecem informações pontuais, utilizando o balanço hídrico no solo ou lisímetro, por exemplo. No entanto, esses métodos costumam ser caros, difíceis de serem trabalhados e só são eficientes quando explorados por especialistas no assunto (NEVES et al., 2007). Nas últimas décadas, com o desenvolvimento dos produtos de sensoriamento remoto, foram desenvolvidas técnicas de estimativa do balanço de radiação na superfície, dos fluxos de calor e da evapotranspiração através de imagens de satélite. A grande vantagem desses métodos é permitir estimativas em escala regional, tornando-se ferramentas de grande utilidade para o manejo racional da irrigação na bacia hidrográfica (SILVA et al., 2005; SILVA \& BEZERRA, 2006).

Um dos algoritmos utilizando técnicas de sensoriamento remoto é o SEBAL (Surface Energy Balance Algorithm for Land), proposto por BASTIAANSSEN (1995). Esse algoritmo faz uso apenas das radiâncias espectrais registradas em sensores de satélites e de um conjunto mínimo de dados meteorológicos que incluem a velocidade do vento e a temperatura do ar para calcular o balanço de energia à superfície da terra e, assim, obter a estimativa da evapotranspiração nas escalas horária e diária (COURAULT et al., 2002). Segundo DI PACE et al. (2008), esse algoritmo leva em consideração a variabilidade espacial de muitos elementos micrometeorológicos, através de funções semiempíricas e pode ser aplicado em diversos agrossistemas. O referido algoritmo é capaz de realizar estimativas precisas da evapotranspiração de áreas agrícolas relativamente horizontais.

Os resultados dos estudos de validação são satisfatórios apesar de o algoritmo SEBAL ser fundamentado em formulações empíricas. BASTIAANSSEN (2000) comparou as estimativas do SEBAL com medições de campo e obteve um erro relativo na fração evaporativa de $20 \%, 10 \%$ e $1 \%$ nas escalas de $1 \mathrm{~km}, 5 \mathrm{~km}$ e $100 \mathrm{~km}$, respectivamente, concluindo que o SEBAL mostra melhores resultados em escala regional, como também em áreas com superfície heterogênea.

Dentro dessa perspectiva, este estudo tem como objetivo estimar a evapotranspiração horária em três regiões do Estado do Ceará (região do Baixo Acaraú, região da Chapada do Apodi e região da Chapada do Araripe), aplicando o algoritmo SEBAL a imagens do satélite Landsat 5, avaliando a variabilidade local e a regional da evapotranspiração.

\section{MATERIAL E MÉTODOS}

\section{Área de estudo}

Na Figura 1, apresentam-se as áreas estudadas em uma composição RGB das bandas IV, III e II (infravermelho próximo, vermelho e verde, respectivamente). A primeira região abrangendo a parte baixa da bacia hidrográfica do Acaraú, onde está localizado o Distrito Irrigado Baixo Acaraú (DIBAU); a segunda, a Chapada do Apodi, identifica a área onde está sitiado o Distrito Irrigado Jaguaribe Apodi (DIJA), e a última área de estudo é representativa da Chapada do Araripe, no Cariri cearense. 


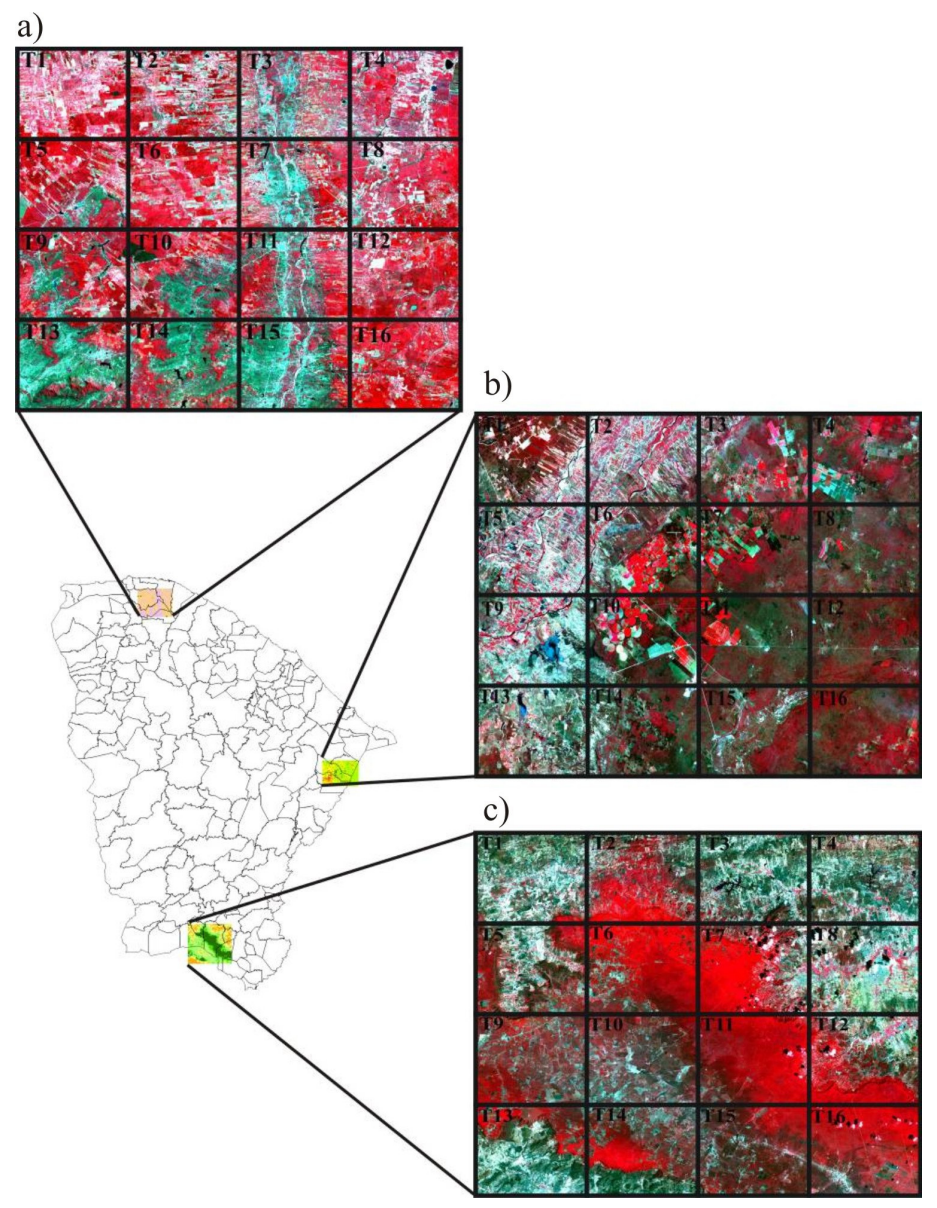

FIGURA 1. Localização das três áreas de estudo no Estado do Ceará: a) Baixo Acaraú; b) Chapada do Apodi, e c) Chapada do Araripe com a representação das sub-regiões utilizadas na análise estatística. Location of three studied areas in the state of Ceará presenting the sub regions used in the statistical analyses.

A maior parte do Estado do Ceará apresenta um clima do tipo tropical quente semiárido, com algumas áreas de clima tropical quente semiárido brando, tropical quente subúmido, tropical quente úmido e tropical subquente úmido (IPECE, 2008). As precipitações pluviométricas apresentam uma distribuição unimodal com $80 \%$ do total concentrados nos quatro primeiros meses do ano.

As três imagens são associadas ao segundo semestre de 2006, no mês de agosto para as regiões do Baixo Aracaú e da Chapada do Apodi e no mês de dezembro para a Região da Chapada do Araripe. O segundo semestre, por ser um período seco no Estado do Ceará, é então uma época em que a irrigação ocorre com maior intensidade. Nesta época do ano, uma estimativa precisa da evapotranspiração faz-se necessária, tendo como objetivo o uso racional da água na irrigação.

Na Figura 2 estão expostas as precipitações mensais para o ano de 2006 provenientes dos Postos pluviométricos (PCDs), coletados junto à Fundação Cearense de Meteorologia e Recursos Hídricos (FUNCEME) localizados nas áreas cobertas pelas três imagens. Na Figura 2, evidencia-se a concentração das precipitações no primeiro semestre, das três regiões estudadas. Nota-se também que as imagens representam o período seco do ano de 2006 (segundo semestre). 


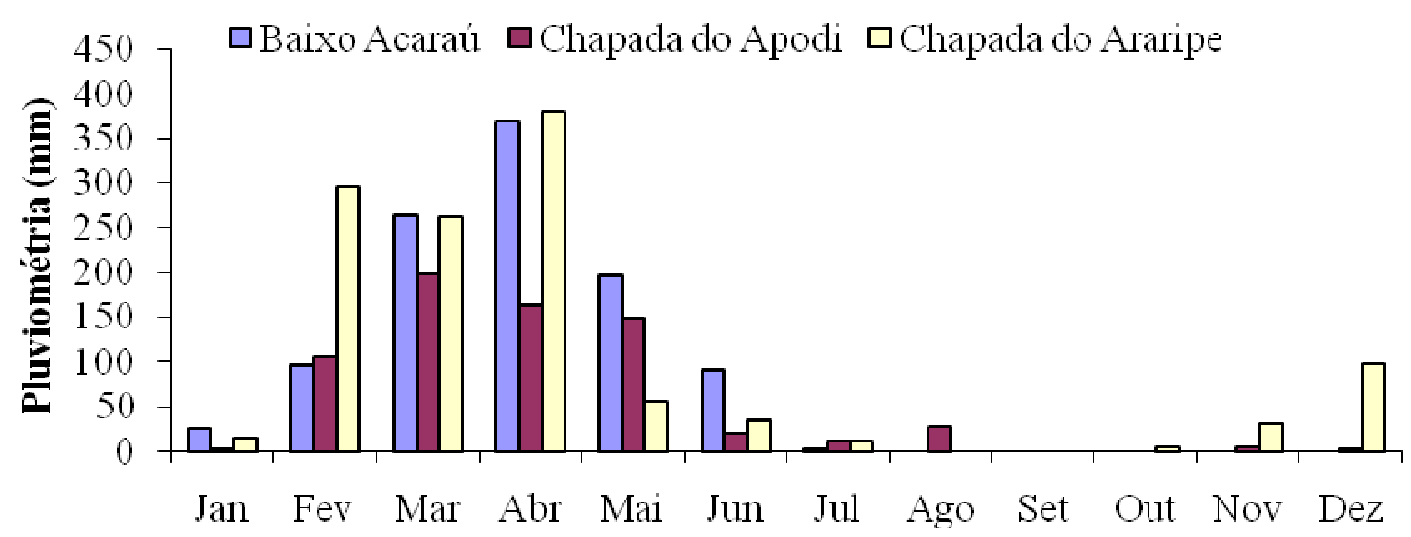

FIGURA 2. Totais mensais da precipitação pluviométrica $(\mathrm{mm})$ do ano de 2006 nas áreas estufadas. Monthly Total of rainfall $(\mathbf{m m})$ for 2006 in the studied areas.

\section{Dados utilizados para aplicação do algoritmo SEBAL}

As observações das bandas I; II; III; IV; V; VI e VII das seguintes imagens do satélite LANDSAT 5-TM adquiridas junto ao Instituto Nacional de Pesquisas Espaciais (INPE): órbita 218, ponto 62 - Imagem referente à região de Acaraú, com passagem do satélite no dia 6-8-2006, no horário de $12 \mathrm{~h} 45 \mathrm{~min} 4 \mathrm{~s}$, órbita 216, ponto 64 - Imagem que cobre a Chapada do Apodi, com passagem do satélite no dia 8-8-2006, às $12 \mathrm{~h} 34 \mathrm{~min} 08 \mathrm{~s}$, órbita 217 e ponto 65 - Imagem referente à região da Chapada do Araripe, com passagem do satélite no dia 21-12-2006, no horário de $12 \mathrm{~h}$ $42 \mathrm{~min} 32 \mathrm{~s}$.

\section{Algoritmo SEBAL}

Neste trabalho, o algoritmo SEBAL foi desenvolvido integralmente no software ArcMap ${ }^{\circledR}$, exceto a etapa do cálculo do fluxo de calor sensível que foi realizada no software Matlab, o qual possibilita a programação do processo iterativo associado.

As imagens foram adquiridas junto ao Instituto Nacional de Pesquisas Espaciais (INPE), cobrindo: (a) a região do Baixo Acaraú (no dia 6-8-2006, às 12h 45min 41s); (b) a região da Chapada do Apodi (no dia 8-8-2006, às $12 \mathrm{~h} 34 \mathrm{~min}$ 08s), e (c) a região da Chapada do Araripe (no dia 21-12-2006, às $12 \mathrm{~h} 42 \mathrm{~min} 32 \mathrm{~s}$ ). A elevação do terreno nas regiões estudadas foi obtida do levantamento da superfície realizado pela Shuttle Radar Topography Mission - SRTM (NASA, 2007). As velocidades do vento foram obtidas nas estações meteorológicas dos municípios do Acaraú, Quixeré e Santana do Cariri, localizadas nas regiões de estudo.

O algoritmo SEBAL calcula a evapotranspiração horária a partir do fluxo de calor latente, este sendo estimado como resíduo do balanço de energia na superfície (Equação. 1):

$$
\mathrm{LE}=\mathrm{R}_{\mathrm{n}}-\mathrm{G}-\mathrm{H}
$$

em que,

LE - fluxo de calor latente, $\mathrm{W} \mathrm{m}^{-2}$;

$\mathrm{R}_{\mathrm{n}}$ - balanço de radiação na superfície, $\mathrm{W} \mathrm{m}^{-2}$;

$\mathrm{G}$ - fluxo de calor no solo, $\mathrm{W} \mathrm{m}^{-2}$, e

$\mathrm{H}$ - fluxo de calor sensível, $\mathrm{W} \mathrm{m}^{-2}$.

Na sequência, explicitam-se os procedimentos principais do algoritmo SEBAL, detalhando-se somente as etapas necessárias à análise dos resultados. Para descrição completa, pode ser consultado o trabalho de BASTIAANSSEN (1995).

Balanço de radiação: A radiação líquida é calculada da seguinte maneira: 


$$
\mathrm{R}_{\mathrm{n}}=(1-\alpha) \mathrm{R}_{\mathrm{s} \downarrow}-\mathrm{R}_{\mathrm{L} \uparrow}+\varepsilon_{\mathrm{o}} \mathrm{R}_{\mathrm{L} \downarrow}
$$

em que,

$\alpha$ - albedo da superfície, adimensional;

$R_{s \downarrow}$ - radiação de onda curta incidente, $\mathrm{W} \mathrm{m}^{-2}$;

$R_{L \uparrow}$ - radiação de onda longa emitida, $\mathrm{W} \mathrm{m}^{-2}$;

$\varepsilon_{o}$ - emissividade da superfície, adimensional, e

$R_{L \downarrow}$ - radiação de onda longa incidente, $\mathrm{W} \mathrm{m}^{-2}$.

A etapa preliminar é a calibração radiométrica das bandas e o cálculo das reflectâncias monocromáticas. Isso permite calcular o albedo no topo da atmosfera. Devido às interações da atmosfera na radiação solar, é necessário realizar correção do albedo no topo da atmosfera, notadamente em função da transmissividade atmosférica, calculada pela equação:

$$
\tau_{\mathrm{sw}}=0,75+210^{-5} \mathrm{z}
$$

em que,

$$
\mathrm{z} \text { - elevação do terreno, } \mathrm{m} \text {. }
$$

Vários autores utilizam um valor médio de $\mathrm{z}$ na região de estudo, ou uma altitude representativa da cena. Neste trabalho, utilizaram-se os dados de elevação do terreno da SRTM, para ter melhor representação dos efeitos do relevo, principalmente nas regiões de serras.

É necessário calcular, também, os índices de vegetação (sem dimensão) seguintes: NDVI (Normalized Difference Vegetation Index), SAVI (Soil Adjusted Vegetation Index) e IAF (Índice de Área Foliar). O NDVI é calculado pela seguinte equação:

$$
\mathrm{NDVI}=\frac{\rho_{\mathrm{IV}}-\rho_{\mathrm{III}}}{\rho_{\mathrm{IV}}+\rho_{\mathrm{III}}}
$$

em que,

$$
\begin{gathered}
\rho_{I V} \text { e } \rho_{I I I} \text { - reflectâncias das bandas IV (infravermelho próximo) e III (vermelho), } \\
\text { respectivamente. }
\end{gathered}
$$

Fluxo de calor no solo: Para a determinação do fluxo de calor no solo (estocado no interior do solo e da vegetação, por condução), calcula-se a razão $G / R_{n}$ : Para pixels em corpos hídricos, ALLEN et al. (2002) recomendam $\mathrm{G} / \mathrm{R}=0,5$, os demais pixels são calculados pela eq.(5):

$$
\frac{\mathrm{G}}{\mathrm{R}_{\mathrm{n}}}=\left[\mathrm{T}_{\mathrm{s}}(0,0038+0,0074 \alpha)\left(1-0,98 \mathrm{NDVI}^{4}\right)\right]
$$

em que,

$\mathrm{G}$ - fluxo de calor no solo, $\mathrm{W} \mathrm{m}^{-2}$;

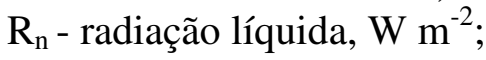

$\mathrm{T}_{\mathrm{s}}$ - temperatura da superfície, ${ }^{\circ} \mathrm{C}$;

$\alpha$ - albedo da superfície, adimensional, e

NDVI - índice de vegetação (adimensional) já citado.

Fluxo de calor sensível: A estimativa do fluxo de calor sensível $(\mathrm{H})$ é a etapa mais complexa do algoritmo SEBAL. Trata-se de um processo iterativo, que foi programado no software Matlab. Nesse processo, considera-se inicialmente que a atmosfera se encontra em condições de estabilidade neutras. Nas demais iterações, correções são incluídas, em funções de condições de estabilidade estimadas. O fluxo de calor sensível é calculado pela equação seguinte: 


$$
H=\frac{\rho C_{p} d T}{r_{a h}}
$$

em que,

$\rho$ - representa a massa específica do ar úmido, $1,15 \mathrm{~kg} \mathrm{~m}^{-3}$;

$\mathrm{C}_{\mathrm{p}}$ - calor específico do ar, $1005 \mathrm{~J} \mathrm{~kg}^{-1} \mathrm{~K}^{-1}$;

dT - diferença de temperatura entre a superfície e a região imediatamente superior, $\mathrm{K}$, e

$\mathrm{r}_{\mathrm{ah}}$ - resistência aerodinâmica ao transporte de calor, $\mathrm{s} \mathrm{m}^{-1}$.

No algoritmo SEBAL, assume-se que dT segue uma variação linear em função da temperatura de superfície:

$$
\mathrm{dT}=\mathrm{a}+\mathrm{bT}_{\mathrm{S}}
$$

Devem ser então escolhidos dois pixels característicos da região de estudo: o "pixel frio", em uma área bem vegetada e irrigada, ou em corpo hídrico, onde se assume que o fluxo de calor sensível é nulo, e o "pixel quente", em uma área de solo nu, seca, onde se considera que o fluxo de calor latente é nulo. Obtém-se um sistema de duas equações, cuja resolução fornece as duas incógnitas $a$ e $b$, e permite obter dT para todos os pixels.

Fluxo de calor latente e evapotranspiração horária: Após o cálculo da radiação líquida e dos fluxos de calor na superfície, deduz-se o fluxo de calor latente pela eq.(1).

A evapotranspiração horária é calculada como:

$$
\mathrm{ET}_{\mathrm{h}}=3600 \frac{\mathrm{LE}}{\rho \lambda}
$$

em que,

LE - fluxo de calor latente, $\mathrm{W} \mathrm{m}^{-2}$;

$\rho$ - massa específica da água, $\mathrm{kg} \mathrm{m}^{-3}$;

$\lambda$ - calor latente de vaporização da água $\left(2,45 \times 10^{6} \mathrm{~J} \mathrm{~kg}^{-1}\right)$, e o coeficiente 3600 vem da conversão de variável instantânea em variável horária.

\section{Análise estatística}

Para detectar uma variabilidade local significativa na evapotranspiração horária, foi aplicado o teste de Tukey, a 5\%, a cada imagem, utilizando as 16 Sub-Regiões definidas na Figura 1. Cada uma dessas Sub-Regiões tinha 90000 pixels. Para estimar a variabilidade regional, as médias de evapotranspiração das três imagens foram comparadas entre si pelo método de Tukey, a 5\% de probabilidade. Essas análises estatísticas foram realizadas com o software SAEG (Sistema para Análise Estatística) versão 9.1.

\section{RESULTADOS E DISCUSSÃO}

\section{Mapas de evapotranspiração horária}

$\mathrm{Na}$ região do Acaraú (Figura 3), os pixels de cor vermelha representam áreas que apresentaram uma taxa de evapotranspiração inferior a $0,25 \mathrm{~mm} \cdot \mathrm{h}^{-1}$, que são áreas de solo exposto e de vegetação nativa bastante rala, correspondendo a $0,61 \%$ da região. As áreas de cor cinza identificam evapotranspiração horária variando de 0,45 a $0,60 \mathrm{~mm} \mathrm{~h}^{-1}$, e equivalem a $34,81 \%$ da região. A vegetação presente nessa área é a Caatinga Rala, que apresentou uma radiação líquida inferior a $500 \mathrm{~W} \mathrm{~m}^{-2}$. SILANS \& SILVA (2007), estudando o fluxo de calor sensível e evapotranspiração na caatinga em São João do Cariri, na Paraíba, obtiveram valores de Radiação Líquida variando de 400 a $500 \mathrm{~W} \mathrm{~m}^{-2}$ em períodos próximos ao meio-dia.

Em torno de 43,06\% da região, a taxa da evapotranspiração variou de 0,60 a $0,75 \mathrm{~mm} \mathrm{~h}^{-1}$, sendo esta área representada pelos pixels verde-claros, a qual corresponde a uma vegetação perene 
de porte elevado, predominantemente, cajueiros. As áreas com ETh variando de 0,75 a $0,98 \mathrm{~mm} \mathrm{~h}^{-1}$ identificam, em sua maioria, corpos hídricos. MENESES (2006), usando o SEBAL no município de Santa Barbara - MG, em setembro de 2003, encontrou uma evapotranspiração variando de 0 a $0,56 \mathrm{~mm} \mathrm{~h}^{-1} ; 0,56$ a $0,73 \mathrm{~mm} \mathrm{~h}^{-1} ; 0,73$ a $0,92 \mathrm{~mm} \mathrm{~h}^{-1}$; em solo com pouca cobertura, um lago e vegetação, respectivamente.

a)

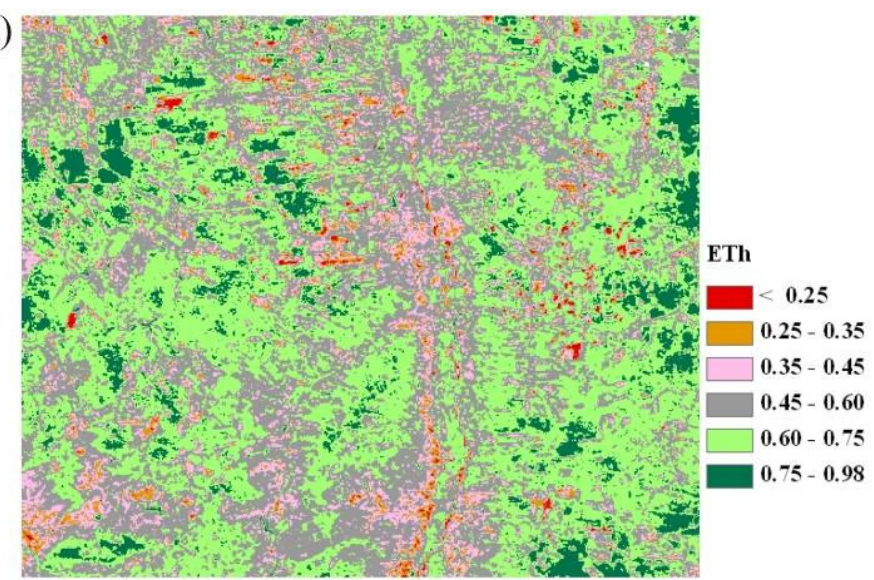

b)

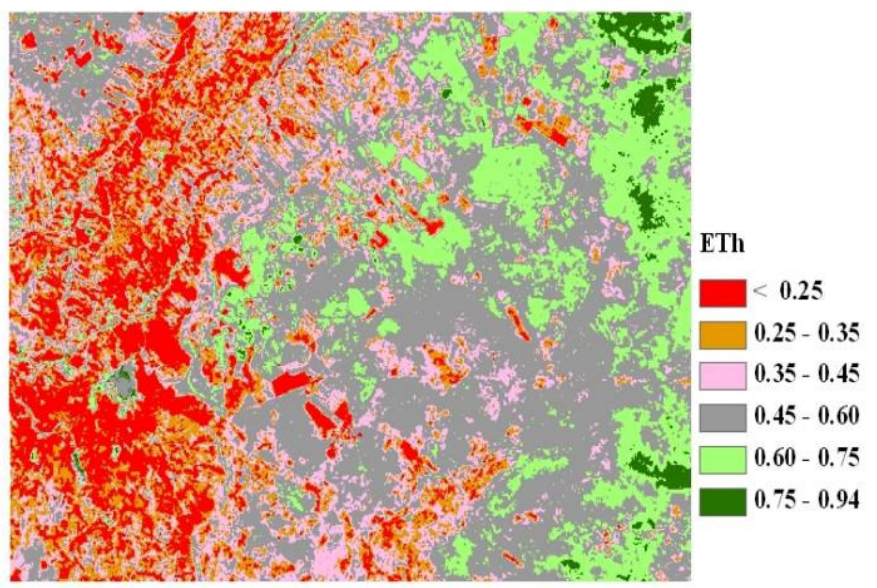

c)

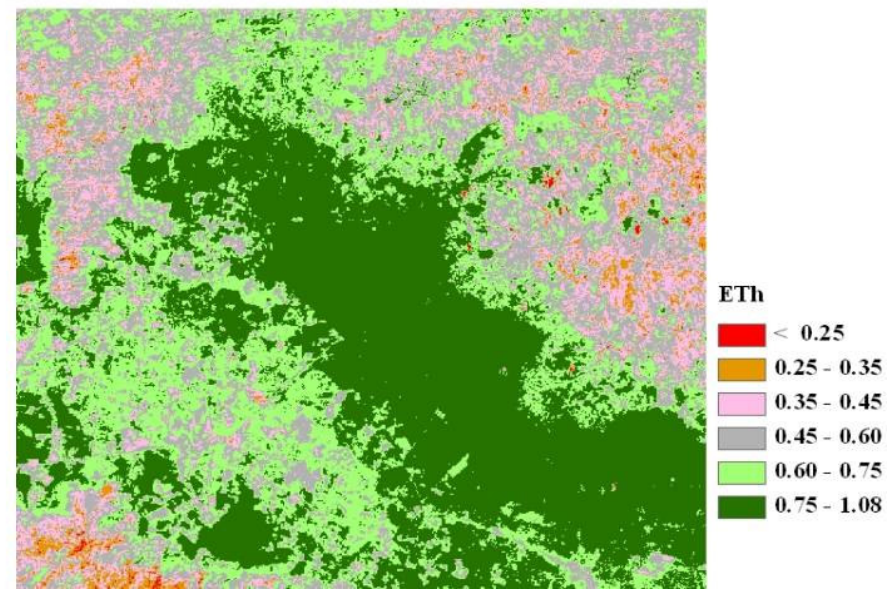

FIGURA 3. Evapotranspiração horária $\left(\mathrm{ET}_{\mathrm{h}} \mathrm{em} \mathrm{mm} \cdot \mathrm{h}^{-1}\right)$ na três regiões de estudo: (a) Baixo Acaraú; (b) Chapada do Apodi, e (c) Chapada do Araripe. Hourly evapotranspiration in the studied areas: (a) Baixo Acaraú, (b) Chapada do Apodi, and (c) Chapada do Araripe.

Na região da Chapada do Apodi (Figura 03b), foram obtidos valores de ETh inferiores a $0,25 \mathrm{~mm} \mathrm{~h}^{-1}$ em $13,62 \%$ da área total e representa solo exposto e vegetação nativa rala próximos ao curso do Rio Quixeré, a qual é identificada na imagem pelos pixels de cor vermelha. Já os pixels de 
cor cinza representam uma evapotranspiração entre 0,45 a $0,60 \mathrm{~mm} \mathrm{~h}^{-1}$ e correspondem a 37,68\% da imagem. Grande parte dessa superfície caracteriza a vegetação nativa que está distribuída em sua maioria sobre as áreas não exploradas com a irrigação na Chapada do Apodi.

As áreas irrigadas do Distrito Irrigado Jaguaribe Apodi tiveram ETh próximo de $0,75 \mathrm{~mm} \mathrm{~h}^{-1}$. A ETh na Lagoa do Sal, no Rio Quixeré e nas áreas de vegetação densa, foi de 0,75 a $0,94 \mathrm{~mm} \mathrm{~h}^{-1}$, corroborando os resultados de DURAND et al. (2007), que obtiveram uma evapotranspiração horária entre 0,65 a $1,00 \mathrm{~mm} \mathrm{~h}^{-1}$ na mesma área.

Em estudo sobre culturas irrigadas no Estado do Novo México, nos Estados Unidos, utilizando dados do sensor MODIS para analisar a distribuição temporal de ET, NAGLER et al. (2005) obtiveram valores de $6 \mathrm{~mm} \cdot \mathrm{dia}^{-1}$ para a cultura do algodão. Já para as áreas de vegetação nativa, os autores supracitados encontraram valores da ordem de 0,35 a $0,50 \mathrm{~mm} \mathrm{~h}^{-1}$.

Nas áreas pesquisadas no presente estudo, foram identificados valores de evapotranspiração horária variando de menos de $0,25 \mathrm{~mm}$ a valores superiores a 0,75 (Tabela 1). Verifica-se que um percentual de área superior a $80 \%$ da Chapada do Apodi expressou uma ETh inferior a 0,60 $\mathrm{mm} \mathrm{h}^{-1}$, o que indica ser uma região mais seca que as outras estudadas.

TABELA 1. Distribuição da evapotranspiração horária nas três regiões estudadas. Hourly evapotranspiration for the three studied regions.

\begin{tabular}{|c|c|c|c|c|c|c|}
\hline \multirow[b]{2}{*}{ Áreas } & \multicolumn{6}{|c|}{$\%$ por Intervalo } \\
\hline & $\begin{array}{l}<0,25 \\
(\mathrm{~mm})\end{array}$ & $\begin{array}{c}0,25 \text { a } 0,35 \\
(\mathrm{~mm})\end{array}$ & $\begin{array}{c}0,35 \text { a } 0,45 \\
(\mathrm{~mm})\end{array}$ & $\begin{array}{c}0,45 \text { a } 0,60 \\
(\mathrm{~mm})\end{array}$ & $\begin{array}{c}0,60 \text { a } 0,75 \\
(\mathrm{~mm})\end{array}$ & $\begin{array}{l}>0,75 \\
(\mathrm{~mm})\end{array}$ \\
\hline Acaraú & 0,61 & 2,94 & 2,96 & 34,81 & 43,06 & 8,62 \\
\hline Apodi & 13,62 & 13,74 & 17,37 & 37,68 & 15,85 & 1,73 \\
\hline Araripe & 0,26 & 3,03 & 9,85 & 23,6 & 27,32 & 35,95 \\
\hline
\end{tabular}

$\mathrm{Na}$ Chapada do Araripe, foram obtidos valores de ETh inferiores a 0,25 $\mathrm{mm} \mathrm{h}^{-1}$ em 0,26\% da região. Em 23,6\% da região, foi registrada uma ETh entre 0,45 e $0,60 \mathrm{~mm} \mathrm{~h}^{-1}$ representada pela cor cinza na Figura 3c, correspondente à vegetação classificada como nativa rala, segundo BRANDÃO et al. (2007). Grande parte dessas áreas expressou um NDVI próximo de 0,30 que pode ser considerado elevado para essa época em uma região semiárida. Uma justificativa para esse fato é a presença da FLONA (Floresta Nacional do Araripe). Nos pixels verde-escuros verificados na Figura 3c, a ETh variou a uma taxa entre 0,75 a $1,08 \mathrm{~mm} \cdot \mathrm{h}^{-1}$,ocupando $35,95 \%$ da região.

BEZERRA (2006), estudando a mesma região, obteve valores superiores a 6,0 $\mathrm{mm} \mathrm{dia}^{-1}$ nas áreas de vegetação densa; já para as áreas próximas a essa vegetação, os valores foram superiores a $5,0 \mathrm{~mm} \mathrm{dia}^{-1}$. Na Tabela 1 da evapotranspiração horária, observa-se que a maior parte da região da Chapada do Araripe detém uma ETh superior a $0,45 \mathrm{~mm} \mathrm{~h}^{-1}$, o que indica ser uma região mais úmida, se comparada às outras duas áreas analisadas.

\section{Análise estatística}

Na Tabela 2, apresenta-se a análise estatística relacionando a evapotranspiração horária para as Sub-Regiões em análise no Baixo Acaraú. Como se pode observar, houve diferença estatística das médias de Evapotranspiração.

A Sub-Região 16 apresentou a maior média de evapotranspiração de toda a imagem, em torno de $0,6513 \mathrm{~mm} \mathrm{~h}^{-1}$. Esse fato explica-se pela maior densidade de vegetação, como pode ser observado na composição RGB da Figura 1. Já sua vizinha, a Sub-Região 15, apresentou a menor média com um valor da ordem de $0,5296 \mathrm{~mm} \mathrm{~h}^{-1}$. Essa é uma Sub-Região que está sobre o rio Acaraú, onde a presença de vegetação ao longo do rio é bem inferior às outras Sub-Regiões. Uma parte do Distrito de Irrigação do Baixo Acaraú está representada na Sub-Região 11, justificando sua maior evapotranspiração em relação às outras Sub-Regiões localizadas ao longo do rio. 
TABELA 2. Média da Evapotranspiração nas Sub-Regiões do Baixo Acaraú. Average hourly evapotranspiration for all sub regions of the Baixo Acaraú.

\begin{tabular}{cc}
\hline Sub-Região & Evapotranspiração ${ }^{*}\left(\mathrm{~mm} \mathrm{~h}^{-1}\right)$ \\
\hline 16 & $0,6513 \mathrm{a}$ \\
12 & $0,6356 \mathrm{ab}$ \\
5 & $0,6347 \mathrm{ab}$ \\
9 & $0,6243 \mathrm{ab}$ \\
10 & $0,6127 \mathrm{abc}$ \\
8 & $0,6124 \mathrm{abc}$ \\
4 & $0,6104 \mathrm{abc}$ \\
6 & $0,6047 \mathrm{abc}$ \\
11 & $0,5896 \mathrm{abc}$ \\
14 & $0,5788 \mathrm{abc}$ \\
1 & $0,5750 \mathrm{abc}$ \\
2 & $0,5594 \mathrm{bc}$ \\
13 & $0,5564 \mathrm{bc}$ \\
3 & $0,5562 \mathrm{bc}$ \\
7 & $0,5475 \mathrm{bc}$ \\
15 & $0,5296 \mathrm{c}$ \\
\hline
\end{tabular}

"Médias seguidas da mesma letra não diferem entre si, pelo teste de Tukey, a 5\% de probabilidade.

Na Tabela 3, mostra-se a análise estatística das Sub-Regiões da Imagem que cobre a Chapada do Apodi. Observa-se que há diferença de 5\% nos valores médios de evapotranspiração.

TABELA 3. Média da Evapotranspiração em todas as Sub-Regiões da Chapada do Apodi. Average hourly evapotranspiration for all sub regions of the Chapada do Apodi.

\begin{tabular}{rc}
\hline Sub-Região & Evapotranspiração ${ }^{*}\left(\mathrm{~mm} \mathrm{~h}^{-1}\right)$ \\
\hline 4 & $0,6077 \mathrm{a}$ \\
8 & $0,5955 \mathrm{a}$ \\
16 & $0,5887 \mathrm{a}$ \\
12 & $0,5532 \mathrm{ab}$ \\
7 & $0,5413 \mathrm{ab}$ \\
3 & $0,5077 \mathrm{abc}$ \\
11 & $0,4901 \mathrm{abcd}$ \\
6 & $0,4467 \mathrm{bcd}$ \\
10 & $0,4421 \mathrm{bcd}$ \\
15 & $0,4411 \mathrm{bcd}$ \\
1 & $0,3938 \mathrm{cde}$ \\
14 & $0,3916 \mathrm{cde}$ \\
2 & $0,3514 \mathrm{de}$ \\
5 & $0,2993 \mathrm{e}$ \\
13 & $0,2914 \mathrm{e}$ \\
9 & $0,2753 \mathrm{e}$
\end{tabular}

* Médias seguidas da mesma letra não diferem entre si, pelo teste de Tukey, a 5\% de probabilidade.

As Sub-Regiões 4; 8; 16 e 12 não apresentaram diferenças estatísticas na evapotranspiração estimada. Como podem ser observadas na Figura 1, essas Sub-Regiões estão localizados sobre a Chapada do Apodi, onde há maior presença de vegetação. DURAND et al. (2007) verificaram um NDVI variando de 0,17 a 0,27 para essa vegetação, podendo a mesma ser classificada como a caatinga mais densa do que aquelas próximas ao Rio Jaguaribe. Essa maior densidade pode ser explicada por uma condição de melhor solo na Chapada do Apodi, como descreve FIALHO et al. (2006). Em uma caatinga parecida com a existente no vale do Rio Jaguaribe, SILVA et al. (2005) 
obtiveram NDVI médio de 0,16 em uma área próxima do Rio São Francisco. A Sub-Região 6, apesar de apresentar uma parte de sua área próxima ao Rio Jaguaribe com vegetação bastante rala e solo exposto, apresentou uma evapotranspiração média de $0,4467 \mathrm{~mm} . \mathrm{h}^{-1}$, considerada intermediária, comparada às outras Sub-Regiões, já que em uma grande parte dessa Sub-Região estão as áreas irrigadas do DIJA. A Sub-Região 9 foi a que apresentou a menor média de evapotranspiração, justificável já que ela é composta por solo exposto e vegetação rala.

$\mathrm{Na}$ Tabela 4, apresenta-se a análise estatística da evapotranspiração horária para as Sub-Regiões da Chapada do Araripe. Como se pode observar, houve diferença estatística das médias de Evapotranspiração.

TABELA 4. Média da evapotranspiração horária em todas as sub-regiões da Chapada do Araripe. Average hourly evapotranspiration for all sub regions of the Chapada do Araripe.

\begin{tabular}{cc}
\hline Sub-Região & Evapotranspiração ${ }^{*}\left(\mathrm{~mm} \mathrm{~h}^{-1}\right)$ \\
\hline 15 & $0,8863 \mathrm{a}$ \\
6 & $0,8839 \mathrm{a}$ \\
11 & $0,8773 \mathrm{a}$ \\
10 & $0,8756 \mathrm{a}$ \\
16 & $0,8357 \mathrm{a}$ \\
5 & $0,8193 \mathrm{a}$ \\
1 & $0,7798 \mathrm{ab}$ \\
14 & $0,7565 \mathrm{abc}$ \\
9 & $0,7245 \mathrm{abcd}$ \\
7 & $0,6953 \mathrm{abcde}$ \\
2 & $0,6258 \mathrm{bcde}$ \\
13 & $0,6257 \mathrm{bcde}$ \\
12 & $0,5828 \mathrm{cde}$ \\
3 & $0,5735 \mathrm{cde}$ \\
4 & $0,5316 \mathrm{de}$ \\
8 & $0,5087 \mathrm{e}$ \\
\hline
\end{tabular}

* Médias seguidas da mesma letra não diferem entre si, pelo teste de Tukey, ao nível de 5\% de probabilidade.

A Sub-Região em que foi observada a menor evapotranspiração foi a 8, onde estão localizadas as cidades do Juazeiro e Crato. As Sub-Regiões 3, 4 e 12 são compostas quase em sua totalidade por caatinga rala, onde também foram registrados baixos valores de evapotranspiração horária. Para essa mesma vegetação, em setembro de 2004, BEZERRA (2006) encontrou valores da taxa de evapotranspiração inferiores a $2 \mathrm{~mm} \mathrm{dia}^{-1}$. As Sub-Regiões $15 ; 6 ; 11 ; 10 ; 16$ e 5 estão quase que totalmente inclusas dentro da área da reserva florestal, com valores de evapotranspiração bem superiores às outras Sub-Regiões. A Sub-Região 7 não apresentou diferença estatística de nenhuma outra. Observando a Figura 1, pode ser constatado que uma parte dela é composta por uma vegetação da reserva, e a outra, por uma vegetação da caatinga, compensando assim os valores, tornando essa Sub-Região intermediária entre as outras. Ainda nessa Sub-Região, foram encontradas algumas nuvens que apresentam albedos superiores a $60 \%$, o que de acordo com ALLEN et al. (2002) é normal, já que superfícies muito brancas podem apresentar albedo em torno de $90 \%$.

A região da Chapada do Araripe apresentou a maior média de evapotranspiração, alcançando valores da ordem de $0,7239 \mathrm{~mm} \mathrm{~h}^{-1}$ (Tabela 5). A região da Chapada do Apodi foi a região de menor média de evapotranspiração, com um valor da ordem de $0,4510 \mathrm{~mm} \mathrm{~h}^{-1}$. A mesma região apresentou o maior coeficiente de variação $(24,55 \%)$ entre os blocos devido à região ser composta por Sub-Regiões extremamente secas e outras terem a presença de áreas irrigadas. A imagem que 
teve as Sub-Regiões com valores de evapotranspiração mais uniforme foi a do Baixo Acaraú, tendo menor coeficiente de variação, com valor da ordem de $6,10 \%$.

TABELA 5. Médias da evapotranspiração horária e coeficientes de variação nas três regiões em estudo. Average hourly evapotranspiration and coefficient of variation for the three studied regions.

\begin{tabular}{lcc}
\hline \multicolumn{1}{c}{ Região } & Médias $\left(\mathrm{mm} \mathrm{h}^{-1}\right)$ & Coeficiente de Variação (\%) \\
\hline Chapada do Araripe & $0,7239 \mathrm{a}$ & 18,54 \\
Baixo Acaraú & $0,5924 \mathrm{~b}$ & 6,10 \\
Chapada do Apodi & $0,451 \mathrm{c}$ & 24,55 \\
\hline
\end{tabular}

${ }^{\mathrm{T}}$ Médias seguidas da mesma letra não diferem entre si, pelo teste de Tukey, ao nível de 5\% de probabilidade.

Os valores médios horários de evapotranspiração regional podem ser considerados relativamente altos se comparados, por exemplo, com os valores obtidos por LIMA et al. (2006), que obtiveram evapotranspiração média de $0,30 \mathrm{~mm} \cdot \mathrm{h}^{-1}$ em alguns dias do mês de junho de 2002, em cultivo de sequeiro do feijão-caupi, na microrregião do Brejo Paraíbano.

\section{CONCLUSÕES}

A análise das médias de evapotranspiração horária, pelo método de Tukey, permitiu evidenciar uma variabilidade significativa local nas três regiões estudadas, bem como regional no Estado do Ceará, devido às condições de solo, clima e vegetação diferenciados.

Além de estimar a evapotranspiração e os termos do balanço de energia na superfície, o algoritmo SEBAL permite detectar a existência de áreas degradadas ou em processo de degradação. Áreas com NDVI muito baixo, albedo elevado e baixa evapotranspiração indicam áreas degradadas, de solo exposto ou pouco vegetadas e secas.

\section{AGRADECIMENTOS}

Ao CNPq e à FUNCAP, por fornecerem apoio financeiro ao primeiro e segundo autores, respectivamente; ao INPE, pelas imagens; ao Laboratório de Geoprocessamento - UFC, pelo suporte técnico, e à FUNCEME, pelos dados de precipitação.

\section{REFERÊNCIAS}

ALLEN, R.G.; PEREIRA, L.S.; RAES, D.; SMITH, M. Crop evapotranspiration - Guidelines for computing crop water requirements. Rome: FAO, 1998. (Irrigation and Drainage, Paper 56)

ALLEN, R.G.; TREZZA, R.; TASUMI, M. Surface energy balance algorithms for land-Advance training and users manual, version 1.0. Idaho: Kimberly, 2002. p.98.

BASTIAANSSEN, W.G.M. Regionalization of surface flux densities and moisture indicators in composite terrain, 1995. 273 f. Tese (Ph.D.) - Wageningem Agricultural University, Wageningen, 1995.

BASTIAANSSEN, W.G.M. SEBAL - based sensible and latent heat fluxes in the irrigated Gediz Basin. Journal of Hydrology, Turkey, n. 229, p.87-100, 2000.

BEZERRA, B.G. Obtenção da evapotranspiração diária no Cariri Cearense utilizando imagens Landsat 5-TM e o algoritmo SEBAL. 2006. 135 f. Dissertação (Mestrado em Meteorologia) Universidade Federal de Campina Grande, Campina Grande, 2006.

BRANDÃO, Z.N.; BEZERRA, M.V.C.; SILVA, B. B. Uso do NDVI para determinação da biomassa na chapada do Araripe. In: SIMPÓSIO BRASILEIRO DE SENSORIAMENTO REMOTO, 2007, Florianópolis. Anais... Florianópolis: INPE, 2007. p.75-81. 
COURAULT, D.; SEGUIM, B.; OLIOSO, A. Review to estimate evapotranspiration from remote sensing data: some examples from the simplified relationship to the use mesoscale atmospheric models. ICID WORKSHOP ON REMOTE SENSING OF ET FOR LARGE REGIONS, 2002, Montpellier.

DI PACE, F.T.; SILVA, B.B. da; SILVA, V.P.R. de; SILVA, S.T.A. da. Mapeamento do saldo de radiação com imagens Landsat 5 e modelo de elevação digital. Revista Brasileira de Engenharia Agrícola e Ambiental, Campina Grande, v.12, n.4, p.385-392, 2008.

DURAND, B.J.; GONDIM, R.S.; SILVA, E.J. da; MOREIRA, L.C.J. TEIXEIRA, A.S. dos. Estimativa da evapotranspiração real utilizando o algoritmo SEBAL e imagens do satélite Landsat 7. In: SIMPÓSIO BRASILEIRO DE RECURSOS HÍDRICOS, 17., 2007, São Paulo. Anais... São Paulo: ABRH, 2007.

FIALHO, J.S.; GOMES, V.F.F.; OLIVEIRA, T.S. de; JÚNIOR, J.M.T. da. Indicadores da qualidade do solo em áreas sob vegetação natural e cultivos de bananeira na Chapada do Apodi-CE. Revista Ciência Agronômica, Fortaleza, v.37, n.3, p.250-257, 2006.

IPECE. INSTITUTO DE PESQUISA E ESTRATÉGIA ECONÔMICA DO CEARÁ. Ceará em números/2006. Disponível em: http://www.ipece.ce.gov.br/atlas/capitulo1/12.htm. Acesso em: 12 fev. 2008

LIMA, J.R.S. de; ANTONINO, A.C.D.; SOARES, W.A. de; SILVA, I.F. de. Estimativa da evapotranspiração do feijão-caupi utilizando o modelo de Penman-Monteith. Irriga, Botucatu, v.11, n.4, p.477-491, 2006.

MENESES, S.J.M.C. Evapotranspiração regional utilizando o SEBAL em condições de relevo montanhoso. 2006. 84 f. Dissertação (Mestrado em Engenharia Agrícola) - Universidade Federal de Viçosa, Viçosa-MG, 2006.

NAGLER, P.L.; SCOTT, R.L.; WESTENBURG, C.; CLEVERLY, J.R.; GLENN, E.P.; HUETE, A.R. Evapotranspiration on western U.S. rives estimated using the Enhanced Vegetation Index from MODIS and data from eddy correlation an Bowen ratio flux towers. Remote Sensing of Environment, 2005. In press.

NASA. SRTM. Disponível em: <http://www2.jpl.nasa.gov/srtm/>. Acesso em 20 ago. 2007.

NEVES, B.V.B.; VERSIANI, B.R.; RODRIGUES, P.C.H. Geoprocessamento como ferramenta no estudo de correlação entre a dinâmica da cobertura vegetal e a evapotranspiração. Revista Brasileira de Recursos Hídricos, Porto Alegre, v.12, n.4, p.87-102, 2007.

PAZ, V.P.S.; TEODORO, R.E.F.; MENDONÇA, F.C. Recursos hídricos, agricultura irrigada e meio ambiente. Revista Brasileira de Engenharia Agrícola e Ambiental, Campina Grande, v.4, n.3, p.465-473, 2000.

PEREIRA, A.R.; VILLA NOVA, N.A.; SEDIYAMA, G.C. Evapotranspiração. Piracicaba: FEALQ, 1997. 183 p.

SCHMIDT, W.; COELHO, R.D.; JACOMAZZI, M.A. Distribuição espacial de pivôs centrais no Brasil: I - Região Sudeste. Revista Brasileira de Engenharia Agrícola e Ambiental, 2004, v.8, n. 23, p.330-333, 2004.

SILANS, A.M.B.P. de; SILVA, F.M. da. Fluxo de calor sensível e evapotranspiração na Caatinga: Estudo Esperimental, Revista Brasileira de Recursos Hídricos, Porto Alegre, v.12, n.1, p.177-188, 2007.

SILVA, B.B. da; BEZERRA, M.V.C. Determinação dos fluxos de calor sensível e latente na superfície utilizando imagens Landsat 5-TM. Revista Brasileira de Agrometeorologia, São José dos Campos, v.14, n.2, p.174-186, 2006.

SILVA, B.B. da; LOPES, G.M.; AZEVEDO, P.V.de. Balanço de radiação em áreas irrigadas utilizando imagens Landsat 5 . TM. Revista Brasileira de Meteorologia, São José dos Campos, v.20, n.2, p.243-252, 2005. 